\title{
Recipient-related predictors of kidney transplantation outcomes in the elderly
}

Hatamizadeh P, Molnar MZ, Streja E, Lertdumrongluk P, Krishnan M, Kovesdy CP, Kalantar-Zadeh K. Recipient-related predictors of kidney transplantation outcomes in the elderly.

Abstract: Background: It is not clear whether in old people with endstage renal disease kidney transplantation is superior to dialysis therapy. Methods: We compared mortality rates between kidney transplant recipients (KTRs) and the general population across different age categories. We also examined patient and allograft survival in 15667 elderly KTRs $(65-<90$ yr old, $36 \%$ female) within three age subgroups $(65-<70,70-<75$, and $\geq 75 \mathrm{yr})$.

Results: The rise in the relative risk of death in older age groups was substantially less in KTRs than in the general population, that is, 1.8 and 2.0 vs. 21.4 and 76.6 in those aged $65-<75$ and $\geq 75 \mathrm{yr}$, respectively, compared with 15 - to $<65$-yr-old people (reference group). In 65 - to $<70-$ yr-old KTRs, obesity (BMI $>30 \mathrm{~kg} / \mathrm{m}^{2}$ ) was associated with $19 \%$ higher risk of graft failure (HR: 1.19 [1.07-1.33], $\mathrm{p}=0.002$ ). Diabetes was a predictor of worse patient survival in all age groups but poorer allograft outcome in the youngest age group $(65-<70 \mathrm{yr}$ old $)$ only. None of the examined risk factors affected allograft outcome in the oldest group ( $\geq 75 \mathrm{yr}$ old) although there was a $49 \%$ lower trend of graft failure in very old Hispanic recipients (HR: 0.51 [0.26-1.01], $\mathrm{p}=0.05$ ).

Conclusions: Kidney transplantation may attenuate the age-associated increase in mortality, and its superior survival gain is most prominent in the oldest recipients ( $\geq 75 \mathrm{yr}$ old). The potential protective effect of kidney transplantation on longevity in the elderly deserves further investigation.

\section{Parta Hatamizadeh ${ }^{\text {a,b }}$, Miklos Z. Molnar $^{\mathrm{a}, \mathrm{c}, \mathrm{d}}$, Elani Streja ${ }^{\mathrm{a}}$, Paungpaga Lertdumrongluk ${ }^{a}$, Mahesh Krishnan ${ }^{\mathrm{e}}$, Csaba P. Kovesdy $\mathbf{f}^{\mathrm{f}, \mathrm{g}}$ and Kamyar Kalantar- Zadeh $^{\mathrm{a}, \mathrm{h}}$}

aHarold Simmons Center for Chronic Disease Research \& Epidemiology, University of California Irvine Medical Center, Irvine, CA, USA, 'bivision of Nephrology, University of Michigan, Ann Arbor, MI, USA, Institute of Pathophysiology, Semmelweis University, Budapest, Hungary, 'Department of Medicine, Division of Nephrology, University Health Network, University of Toronto, Toronto, ON, Canada, 'DaVita, Inc., Denver, CO, USA, fDivision of Nephrology, Memphis VA Medical Center, Memphis, TN, USA, ${ }^{9}$ Division of Nephrology, University of Tennessee Health Science Center, Memphis, TN, USA and ${ }^{\text {h}}$ Division of Nephrology and Hypertension, University of California Irvine Medical Center, Orange, CA, USA

Key words: elderly - graft failure - kidney transplantation - longevity - mortality - senior

\author{
Corresponding author: Kamyar Kalantar- \\ Zadeh, MD, MPH, PHD, Harold Simmons \\ Program for Kidney Disease Research \& \\ Epidemiology, Division of Nephrology \& \\ Hypertension, School of Medicine, University \\ of California Irvine, 101 The City Drive South, \\ City Tower, Suite 400 - ZOT: 4088, Orange, CA \\ 92868-3217, USA. \\ Tel.: 714456 5142; fax: 714456 6034; \\ e-mail:kkz@uci.edu
}

Conflict of Interest: Dr. Krishnan is an employee of DaVita. Dr. Kalantar-Zadeh was the medical director of DaVita Harbor-UCLA/ MFI in Long Beach, CA. Other authors have not declared any conflict of interest.

Accepted for publication 13 January 2013
The senior population, aged 65 or older, is increasing rapidly all over the world including the United States (1). According to the National Health and Nutrition Examination Survey (NHANES), the percentage of CKD stage 3 or
4 patients in population aged 60 and above increased from $1.3 \%(1988-1994)$ to $2.3 \%$ (2003-2006) (2). This finding corresponds to a rise in the number of senior kidney transplant recipients. 
Several studies demonstrated a survival advantage with transplantation among the senior patients compared with dialysis patients (3-7). The study by Wolfe and colleagues demonstrated that primary deceased donor transplantation, compared with maintenance hemodialysis, was associated with increased cumulative survival rate after the first year post-transplantation, with an increased projected life span of five yr for patients aged 60-74 yr without diabetes and three yr for the same age group patients with diabetes (7). In a study by Gill and colleagues, the expected survival rates for kidney transplant waitlisted patients aged $\geq 70 \mathrm{yr}$ were $4.5 \mathrm{yr}$ and $8.2 \mathrm{yr}$ for those who received a kidney transplant (8). Senior patients also have a good quality of life after kidney transplantation (9) and lower rates of acute and chronic rejections compared with younger recipients (10). However, the senior KTR survival at one, five, and $10 \mathrm{yr}$ is approximately $80-90,70$, and $50 \%$, respectively (11-19). Given the rapid growth of the number of senior patients undergoing kidney transplantation, it is important to be able to identify the appropriate senior candidates for kidney transplantation.

Published studies regarding the recipient factors that would predict outcomes in senior KTRs are scarce. In a recent study by Heldal et al., Charlson comorbidity index (CCI) scores could not predict mortality in patients aged $75 \mathrm{yr}$ or older who had received their first transplanted kidney. However, CCI scores could do so in first KTRs of both age groups 60-69 and 45-54 yr (20). Wu and colleagues found that a modified CCI score excluding age was a predictor of patient survival in recipients aged $60 \mathrm{yr}$ or older except in the subgroup of these patients who received kidneys from living donors (21).

In the present study, we examined the effects of various recipient-related factors on patient survival and kidney graft outcomes separately in different age groups of senior recipients. We also compared all-cause mortality rates among different age groups in the KTRs with those of the general population to compare age-induced increase in mortality risk in patients with and without kidney transplantation.

\section{Patients and methods}

Patients

The study population consisted of KTRs listed in the SRTR from 2001 until June 2007. The SRTR data system includes data on all transplant donors, wait-listed candidates, and transplant recipients in the United States, which are submitted by members of the Organ Procurement and Transplantation Network (OPTN). The Health Resources and Services Administration (HRSA), U.S. Department of Health and Human Services provides oversight to the activities of the OPTN and SRTR contractors.

This study was approved by the Institutional Review Committees of the Los Angeles Biomedical Research Institute at Harbor-UCLA Medical Center. Because of the large sample size, the anonymity of the studied patients, and the nonintrusive nature of the research, the requirement for informed consent was waived.

Clinical, demographic, and laboratory measures

Demographic data and details of medical history were collected, including age, gender, race, ethnicity, and dialysis vintage. Dialysis vintage was defined as the duration of time between the first day of dialysis treatment and the day of kidney transplantation. Information on the recipients' serum creatinine, serum albumin, weight and height (for calculation of body mass index (BMI)) and seven comorbidities, diabetes, angina (as an indicator of coronary artery disease), chronic obstructive pulmonary disease, hypertension, peptic ulcer, peripheral vascular disease, and cerebrovascular disease, was also collected. BMI $>30 \mathrm{~kg} / \mathrm{m}^{2}$ was considered obese and was analyzed as a dichotomous variable. We analyzed data on senior KTRs, defined as those aged $65 \mathrm{yr}$ or older at the time of first transplantation. This study population was divided into three age groups $(65-<70,70-<75$, and $\geq 75 \mathrm{yr}$ ) for subgroup analyses.

\section{Statistical Methods}

Descriptive results are reported as mean ( \pm standard deviation $[\mathrm{SD}])$, percentage $(\%)$, median, and percentile when appropriate.

We compared the mortality rates and ratios across four age groups $(15-<65,65-<70,70-<75$, and $\geq 75 \mathrm{yr}$ old) between the kidney transplant recipients (derived from the SRTR 2001-2007) and the general population using United States population in 2005 (derived from the National Vital Statistics System). After calculating the crude mortality rates in the KTRs and the general population across three age groups, the relative risk (RR) of death was calculated for the two senior groups and the 15- to $<65$-yr-old group (reference), and the ratio of mortality RR for kidney transplant patients to the general population was estimated in each group. 
For survival analysis within our study population, we used Cox proportional hazard regression models separately across the different age groups. Our two primary outcomes were patient survival and kidney allograft survival. For graft survival analysis, graft failure was defined as re-initiation of dialysis treatment or retransplantation. Imminent graft failure was defined as an estimated glomerular filtration rate (eGFR) $<30 \mathrm{~mL} / \mathrm{min} / 1.73 \mathrm{~m}^{2}$ using the modified diet in renal disease (MDRD) equation, provided there was no eGFR $>40 \mathrm{~mL} /$ $\min / 1.73 \mathrm{~m}^{2}$ reported afterward, to exclude cases of acute rejection or acute kidney injury that had been treated successfully.

We used two different approaches for graft survival analysis. In death-censored graft survival analysis, patients who died before re-initiation of dialysis treatment or retransplantation were censored regardless of the function of their transplanted kidney at the time of death. In combined graft failure-imminent graft failure analysis, patients were followed until either graft failure or imminent graft failure or until censoring (death or end of follow-up period), whichever happened first.

For each analysis, 2 models were examined:

I. Unadjusted model.

II. Adjusted models that included age, gender, race-ethnicity (African Americans and other self-categorized blacks, Caucasian [or non-Hispanic whites], Asians, and Hispanics), dialysis vintage, comorbidities, and pre-transplant parameters including serum creatinine, serum albumin, and BMI.

All analyses were carried out by SAS, version 9.1, SAS Institute Inc., Cary, NC, USA.

\section{Results}

In the comparison of KTRs with the general population, even though KTRs aged $65-<75$ and $\geq 75 \mathrm{yr}$ had 1.8 and 2.0 times higher mortality risk, respectively, compared with those aged 15$<65 \mathrm{yr}$, the age-related rise in mortality was substantially lower among KTRs as opposed to the general population (Fig. 1). The rise in the RR of death in older age groups in the general population was 21.4 and 76.6 in those aged $65-<75$ and $\geq 75 \mathrm{yr}$, respectively, compared with 15 - to $<65$ yr-old people (reference group); hence, there was $75 \%$ and $92 \%$ lower death risk ratio of KTRs to general population in these two elderly age groups (see Fig. 1 and Table S1).

Within the study population, additional analysis was performed on 15667 KTRs older than $65 \mathrm{yr}$

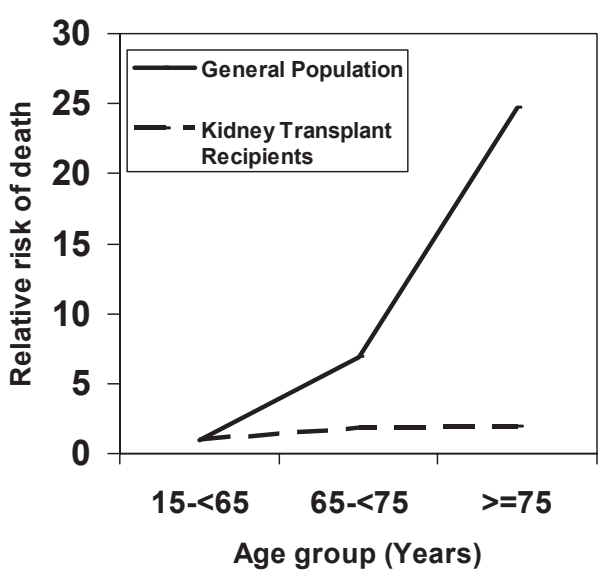

Fig. 1. Relative risk of death with advancing age in the kidney transplant recipients and the general population.

at the time of transplantation. The median followup time for all-cause mortality and graft failure was $1425 \mathrm{~d}$ and that of combined graft failureimminent graft failure was $1242 \mathrm{~d}$. There were 24901 deaths (17\%), 20614 graft failures (14\%) and 21600 imminent graft failures $(15 \%)$, some of whom eventually developed graft failure within the study period. Accordingly, there were a total of 34438 combined graft failure-imminent graft failures $(24 \%)$. Patients' ages ranged between 65 and $90 \mathrm{yr}$, and the number of cases decreased with increasing age. The majority $(64 \%)$ of the patients were in the youngest age group (65-70 yr), with only $8 \%$ older than $70 \mathrm{yr}$ (Table 1 and Fig. S1). Table 1 presents the clinical, demographic, laboratory, and transplant data across four different age groups of KTRs, including the three study age groups as well as adults aged $<65 \mathrm{yr}$ in one group for comparison. The majority of transplant recipients were Caucasians and men in all age groups, particularly in the older groups. In contrast to the increase in crude mortality with advancing age, the combined graft failure-imminent graft failure rate was lower in seniors compared with non-senior recipients and was the lowest in the eldest group. Among the three senior age groups, the incidence of diabetes was lower in the older age groups.

Recipient-related predictors of all-cause mortality in different senior age groups are demonstrated in Table 2 and Fig. 2. Diabetes was a strong predictor of all-cause mortality in all three elderly groups both in unadjusted and adjusted analyses. Female gender was associated with lower all-cause mortality in age groups $65-<70$ yr. However, gender did not show significant association with patient survival in the eldest age group ( $>75 \mathrm{yr})$. In 10101 patients aged 65-<70 yr, Asian and Hispanic races were predictors of lower mortality, and 
Table 1. Incremental categories of recipient age in 145470 kidney-transplanted patients including selected clinical and laboratory values in each group

\begin{tabular}{|c|c|c|c|c|c|c|}
\hline Age range (yr) & All & $15-<65$ & $65-<70$ & $70-<75$ & $\geq 75$ & $\mathrm{p}$-Value \\
\hline Number & 145470 & 129803 & 10101 & 4271 & 1295 & $<0.001$ \\
\hline Age $(y r)($ mean \pm SD) & $47.3 \pm 13.6$ & $44.7 \pm 12.0$ & $66.8 \pm 1.4$ & $71.6 \pm 1.4$ & $77.1 \pm 2.2$ & $<0.001$ \\
\hline Gender (\% women) & 40 & 41 & 38 & 34 & 27 & $<0.001$ \\
\hline Race (\% Caucasian) & 59 & 58 & 64 & 71 & 79 & $<0.001$ \\
\hline Race (\% Hispanic) & 12 & 12 & 10 & 9 & 6 & $<0.001$ \\
\hline Race (\% Asian) & 4 & 4 & 4 & 4 & 3 & 0.22 \\
\hline Race (\% Black) & 23 & 24 & 20 & 16 & 11 & $<0.001$ \\
\hline $\mathrm{BMI}\left(\mathrm{kg} / \mathrm{m}^{2}\right)($ mean $\pm \mathrm{SD})$ & $26.8 \pm 5.5$ & $26.8 \pm 5.6$ & $27.4 \pm 4.9$ & $26.8 \pm 4.5$ & $26.4 \pm 4.4$ & $<0.001$ \\
\hline Deaths (n) (Crude death rate \%) & $24901(17)$ & $20567(16)$ & $2759(27)$ & $1178(28)$ & $397(31)$ & $<0.001$ \\
\hline Graft failure (n) (Crude graft failure rate \%) & $20614(14)$ & $19077(15)$ & $1014(10)$ & $397(9)$ & $126(10)$ & $<0.001$ \\
\hline $\begin{array}{l}\text { Imminent graft failure (n) (Crude imminent } \\
\text { graft failure rate \%) }\end{array}$ & $21600(15)$ & $19598(15)$ & $1306(13)$ & $574(13)$ & $122(9)$ & $<0.001$ \\
\hline $\begin{array}{l}\text { Combined graft failure-Imminent graft failure } \\
\text { (n) (Crude combined graft failure rate \%) }\end{array}$ & $34438(23.67)$ & $31315(24.13)$ & $2027(20.07)$ & $867(20.3)$ & $229(17.68)$ & $<0.001$ \\
\hline Acute rejection episode (n) NEW & 10121 & 9174 & 600 & 256 & 91 & $<0.001$ \\
\hline Acute rejection episode ( $n$ ) & 7306 & 6702 & 365 & 180 & 59 & $<0.001$ \\
\hline \multicolumn{7}{|l|}{ Comorbidities } \\
\hline Diabetes mellitus (\%) & 22 & 21 & 30 & 24 & 16 & $<0.001$ \\
\hline Hypertension (\%) & 77 & 76 & 82 & 82 & 84 & $<0.001$ \\
\hline Angina (\%) & 11 & 9 & 20 & 19 & 19 & $<0.001$ \\
\hline Cerebrovascular disease (\%) & 2 & 2 & 4 & 4 & 3 & $<0.001$ \\
\hline Peripheral vascular disease (\%) & 4 & 4 & 6 & 6 & 4 & $<0.001$ \\
\hline COPD $(\%)$ & 1 & 1 & 2 & 2 & 1 & $<0.001$ \\
\hline Peptic ulcer (\%) & 5 & 5 & 6 & 6 & 6 & $<0.001$ \\
\hline Cancer & 4 & 3 & 8 & 11 & 12 & $<0.001$ \\
\hline \multicolumn{7}{|l|}{ Laboratory values } \\
\hline $\begin{array}{l}\text { Baseline serum creatinine }(\mathrm{mg} / \mathrm{dL}) \\
(\text { mean } \pm \mathrm{SD})\end{array}$ & $8.13 \pm 3.39$ & $8.29 \pm 3.45$ & $7.02 \pm 2.71$ & $6.89 \pm 2.57$ & $6.61 \pm 2.49$ & $<0.001$ \\
\hline Baseline serum albumin $(\mathrm{g} / \mathrm{dL})($ mean $\pm \mathrm{SD})$ & $3.86 \pm 0.66$ & $3.86 \pm 0.67$ & $3.84 \pm 0.59$ & $3.86 \pm 0.60$ & $3.90 \pm 0.52$ & 0.04 \\
\hline PRA (\%) mean (median, 25th-75th percentile) & $13.7(0,0,11)$ & $13.9(11,0,60)$ & $12.4(10,0,49)$ & $11.1(9,0,41)$ & $9.7(7,0.33)$ & $<0.001$ \\
\hline PRA $=0 \%(n)(\%)$ & $72432(50)$ & $64232(50)$ & $5209(52)$ & $2266(53)$ & $725(56)$ & $<0.001$ \\
\hline
\end{tabular}

The study population included the last three groups only. Non-elderly recipients' information is included for comparison. Graft failure: Re-initiation of dialysis treatment or retransplantation. Imminent graft failure: estimated glomerular filtration rate (eGFR) $<30 \mathrm{~mL} / \mathrm{min} / 1.73 \mathrm{~m}^{2}$ using modified diet in renal disease (MDRD) equation, provided there was no eGFR $>40 \mathrm{~mL} / \mathrm{min} / 1.73 \mathrm{~m}^{2}$ reported afterward.

$\mathrm{BMI}$, body mass index; COPD, chronic obstructive pulmonary disease; PRA, panel reactive antibody (last value prior to transplant).

black race was associated with a higher all-cause mortality in the adjusted model. Angina was also a predictor of higher mortality in this age group. However, although obesity was associated with a higher mortality in this age group in the unadjusted model, it was not a predictor of mortality after the adjustment. In 4271 patients aged 70$<75 \mathrm{yr}$, in addition to diabetes and female gender, Hispanic race was a predictor of lower all-cause mortality, whereas black race and angina were predictors of higher all-cause mortality. Interestingly enough, only in transplant recipient patients aged $75 \mathrm{yr}$ or older, obesity was a predictor of all-cause mortality after adjustment.

Fig. 3 and Tables S2 through S4 display recipient-related predictors of graft failure in the three elderly subgroups by two different approaches: the conventional death-censored graft failure and the combined graft failure-imminent graft failure approach. As demonstrated in Fig. 3 and Table
S2, predictors of graft survival, as evaluated by combined graft failure-imminent graft failure approach, are similar to those of patient survival in the youngest elderly group $(65-<70 \mathrm{yr}$ old $)$ with the exception of Asian race and angina, which were not found to be predictors of graft survival in this age group. Additionally, female gender, which was a protective factor against all-cause mortality, was paradoxically associated with worse kidney transplant outcomes. Hispanic race was associated with a better graft survival, and black race, obesity and diabetes were associated with a higher combined graft failure-imminent graft failure in this age group. Indeed in 65- to $<70$-yr-old KTRs, obesity $\left(\right.$ BMI $\left.>30 \mathrm{~kg} / \mathrm{m}^{2}\right)$ was associated with $19 \%$ higher risk of graft failure (adjusted HR: 1.19 [1.07-1.33], $\mathrm{p}=0.002$ ). With conventional death-censored graft survival analysis, however, obesity, diabetes, and gender were not shown to be independent predictors of graft survival in this age subgroup. 
Table 2. Hazard ratios (95\% confidence intervals) of all-cause mortality using Cox regression analyses

\begin{tabular}{|c|c|c|c|c|}
\hline & \multicolumn{2}{|l|}{ Unadjusted } & \multicolumn{2}{|l|}{ Adjusted } \\
\hline & $\mathrm{HR}(95 \% \mathrm{Cl})$ & p-Value & $\mathrm{HR}(95 \% \mathrm{Cl})$ & p-Value \\
\hline \multicolumn{5}{|l|}{ Age $65-<70$ yr $(\mathrm{N}=10$ 101) } \\
\hline Female vs. male recipient (ref.) & $0.86(0.79-0.93)$ & $<0.001$ & $0.88(0.81-0.95)$ & 0.001 \\
\hline Recipient Race- Caucasian & $1.00(0.92-1.08)$ & 0.93 & $1.07(0.98-1.17)$ & 0.11 \\
\hline Recipient Race- Hispanic & $0.91(0.80-1.04)$ & 0.16 & $0.83(0.73-0.95)$ & 0.006 \\
\hline Recipient Race- Asian & $0.64(0.51-0.80)$ & $<0.001$ & $0.63(0.50-0.80)$ & $<0.001$ \\
\hline Recipient Race- Black & $1.18(1.07-1.30)$ & $<0.001$ & $1.16(1.05-1.28)$ & 0.004 \\
\hline BMI $\left(\mathrm{kg} / \mathrm{m}^{2}\right)(>30$ vs. $\leq 30$ [ref.]) & $1.14(1.03-1.25)$ & 0.01 & $1.03(0.93-1.14)$ & 0.53 \\
\hline Diabetic vs. non-diabetic (ref.) & $1.49(1.38-1.62)$ & $<0.001$ & $1.45(1.33-1.57)$ & $<0.001$ \\
\hline Hypertensive vs. non-hypertensive (ref.) & $0.97(0.88-1.70)$ & 0.55 & $0.93(0.84-1.03)$ & 0.14 \\
\hline Angina vs. non-angina (ref.) & $1.32(1.21-1.44)$ & $<0.001$ & $1.20(1.09-1.31)$ & $<0.001$ \\
\hline \multicolumn{5}{|l|}{ Age $70-<75$ yr $(N=4271)$} \\
\hline Female vs. male recipient (ref.) & $0.81(0.71-0.91)$ & $<0.001$ & $0.78(0.69-0.89)$ & $<0.001$ \\
\hline Recipient Race- Caucasian & $0.92(0.81-1.04)$ & 0.19 & $0.97(0.84-1.11)$ & 0.64 \\
\hline Recipient Race- Hispanic & $0.78(0.62-0.99)$ & 0.04 & $0.73(0.57-0.92)$ & 0.008 \\
\hline Recipient Race- Asian & $0.90(0.64-1.25)$ & 0.52 & $0.89(0.63-1.24)$ & 0.48 \\
\hline Recipient Race- Black & $1.36(1.17-1.59)$ & $<0.001$ & $1.35(1.14-1.59)$ & $<0.001$ \\
\hline BMI $\left(\mathrm{kg} / \mathrm{m}^{2}\right)(>30$ vs. $\leq 30$ [ref.]) & $1.16(0.99-1.36)$ & 0.07 & $1.06(0.90-1.26)$ & 0.46 \\
\hline Diabetic vs. non-diabetic (ref.) & $1.453(1.28-1.65)$ & $<0.001$ & $1.32(1.16-1.51)$ & $<0.001$ \\
\hline Hypertensive vs. non-hypertensive (ref.) & $1.058(0.91-1.23)$ & 0.46 & $1.00(0.86-1.16)$ & 1.00 \\
\hline Angina vs. non-Angina (ref.) & $1.303(1.14-1.50)$ & $<0.001$ & $1.17(1.01-1.36)$ & 0.04 \\
\hline \multicolumn{5}{|l|}{ Age $\geq 75$ yr $(N=1295)$} \\
\hline Female vs. male recipient (ref.) & $0.91(1.072-1.15)$ & 0.42 & $0.86(0.67-1.09)$ & 0.21 \\
\hline Recipient Race-Caucasian & $1.14(0.88-1.48)$ & 0.33 & $1.21(0.92-1.59)$ & 0.18 \\
\hline Recipient Race-Hispanic & $0.71(0.45-1.12)$ & 0.14 & $0.68(0.43-1.08)$ & 0.10 \\
\hline Recipient Race-Asian & $0.69(0.34-1.42)$ & 0.32 & $0.63(0.30-1.31)$ & 0.22 \\
\hline Recipient Race-Black & $1.09(0.78-1.54)$ & 0.61 & $1.07(0.75-1.54)$ & 0.70 \\
\hline BMI $\left(\mathrm{kg} / \mathrm{m}^{2}\right)(>30$ vs. $\leq 30$ [ref.]) & $1.61(1.18-2.20)$ & 0.003 & $1.50(1.09-2.07)$ & 0.01 \\
\hline Diabetic vs. non-diabetic (ref.) & $1.38(1.09-1.77)$ & 0.009 & $1.39(1.08-1.79)$ & 0.01 \\
\hline Hypertensive vs. non-hypertensive (ref.) & $1.15(0.87-1.53)$ & 0.3242 & $1.17(0.88-1.57)$ & 0.2737 \\
\hline Angina vs. non-Angina (ref.) & $1.023(0.80-1.31)$ & 0.8603 & $0.95(0.73-1.24)$ & 0.7015 \\
\hline
\end{tabular}

BMI, body mass index; HR, hazard ratio; $\mathrm{Cl}$, confidence interval; Ref, reference group.

In the 70- to 75-yr-old KTRs, only female gender was found to be a predictor of poorer graft survival using the combined approach. The

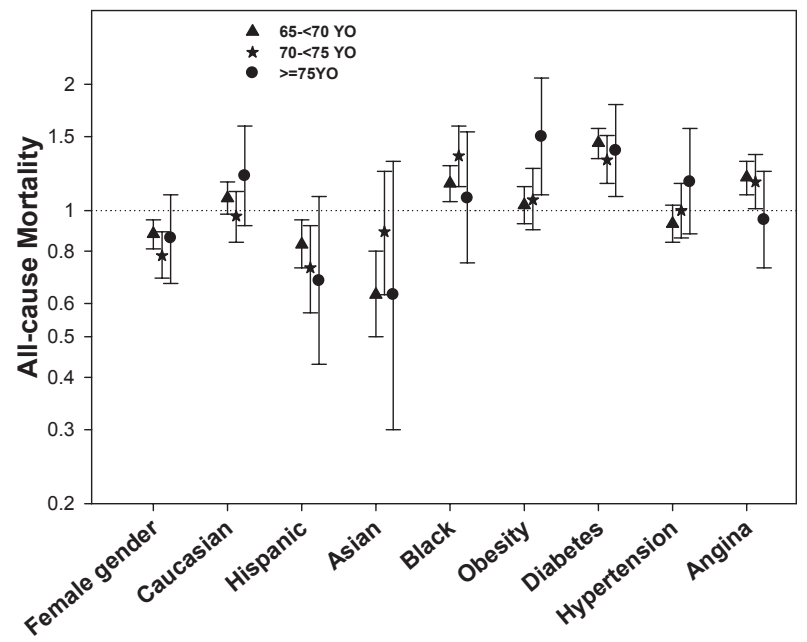

Pretransplant parameters

Fig. 2. Hazard ratio with $95 \%$ confidence interval of all-cause mortality for pre-transplant parameters in three elderly age categories measured by the adjusted model. conventional approach was unable to reveal this association (Table S3). None of the studied parameters were shown to be a predictor of graft survival in the kidney transplant recipients older than $75 \mathrm{yr}$ in adjusted or unadjusted analyses by combined method. Nevertheless, the conventional deathcensored analysis demonstrated a better graft survival for Hispanic patients in this age subgroup (Table S4). In the oldest KTRs ( $>75 \mathrm{yr}$ ), there was a $49 \%$ lower trend of combined graft failure in the Hispanic recipients (HR: 0.51 [0.26-1.01], $\mathrm{p}=0.05$ ).

\section{Discussion}

Our study showed the following findings: (i) Increase in all-cause mortality with advancing age is dramatically attenuated in kidney transplant recipients compared with general population. (ii) Kidney transplant outcome is better in the older than in younger KTRs and is the best in the eldest people (>75 yr old). (iii) Among senior KTRs, diabetes has a negative effect on patient survival in all patients but a negative effect on graft survival only 


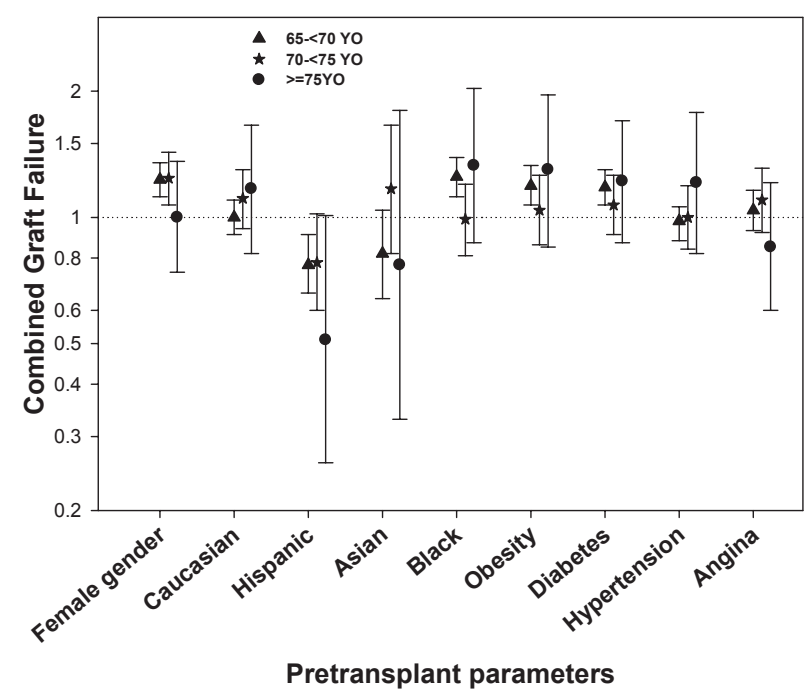

Fig. 3. Hazard ratio with $95 \%$ confidence interval of combined graft failure-imminent graft failure for pre-transplant parameters in three elderly age categories measured by the adjusted model.

in those aged between 65 and $<70$ yr. (iv) Female senior KTRs generally survive better than their male counterparts, but their transplanted kidneys survive less. (v) Obesity in the 65- to 70-yr-old recipients is associated with higher graft failure. (vi) In the very old recipients ( $>75 \mathrm{yr}$ ), Hispanics tend to gain the best outcomes.

The remarkable reduction in all-cause mortality with advancing age in KTRs compared with general population deserves special attention. Further study in this regard can potentially open a new avenue for the study of longevity in addition to the information it can provide about the possible ways to improve kidney transplantation outcomes. It also underscores the fact that kidney transplantation needs to be encouraged in appropriate older patients. Of note, however, potential effects of a selection bias needs to be considered. Older patients who are selected for kidney transplantation may be those who are relatively healthier compared to age-matched patients, whereas younger transplant candidates may not have the same advantage. Another potential explanation of this observation is that the lower increased mortality risk in older transplant recipients is related to a higher mortality in younger transplant recipients, relative to the general population within the same age group. Further studies are needed in this regard.

In a recent study by Tillius and colleagues on 108188 recipients of deceased donor kidneys between 1995 and 2008, older recipients had a better graft survival compared with younger KTRs even with poorer quality of transplanted kidneys
(22). Our study revealed that after including imminent graft failure in the analysis, the rate of combined graft failure-imminent graft failure is less in seniors and is the least in the eldest subgroup. This further emphasizes a potential desirable effect of advanced age on transplant survival, even after eliminating the effect of a potential bias imposed by conventional analysis due to overlooking graft failures in those who died. Therefore, the lower number of older recipients (as shown in Fig. S1) should be revisited, and kidney transplantation of those in the older subgroup should be encouraged for appropriate cases.

The current study also displayed the deleterious effect of diabetes on patient survival in all senior age groups. Even though it seems to be expected, some smaller-scale studies were unable to show this effect (20). Our study also demonstrated the harmful effect of diabetes on graft survival in the senior patients aged 65 to $<70 \mathrm{yr}$ but not in those aged $70 \mathrm{yr}$ or older. Interestingly, even though diabetes is more prevalent among senior recipients compared with younger individuals, within the senior recipients, its prevalence decreases by increasing age. This could be due to the fact that diabetic patients with very advanced age have less chance of being selected for transplantation. This is despite the existing evidence of improved survival with kidney transplantation compared with other renal replacement therapy (RRT) modalities even in the diabetic senior population (7). A newly published study of diabetic kidney transplant recipients showed a decreased post-transplant survival in those who had a poor pre-transplant glycemic control; however, it did not show any effect of pre-transplant glycemic control on allograft outcomes (23). Considering all of these findings, one should be cautious about kidney transplantation in younger groups of senior diabetic patients, particularly if they had a poor glycemic control. Notwithstanding, diabetes per se should not be a contraindication for kidney transplantation in the seniors, especially in those who are aged $70 \mathrm{yr}$ or older. However, this result might be inaccurate due to small sample size. Further studies are needed.

In patients aged between 65 and $<75 \mathrm{yr}$, graft survival was shorter among women compared with men. Nonetheless, women survived longer. This could be due to the fact that women in general have a better life expectancy than men (24) but senior women may get less of a benefit from a kidney transplant compared with men of the same age.

Angina, as an indicator of coronary artery disease, also had no association with graft survival. 
Therefore, angina per se, should not keep the clinician from considering kidney transplantation.

Hispanic recipients generally had a better outcome and African Americans had a worse outcome, particularly in the younger senior age groups. Similar findings have been reported in other populations (25). Putting this finding into consideration will help better selection of the appropriate cases for kidney transplantation.

In general, patient and graft survival in the oldest group demonstrated less association with any of the studied parameters. This could be due to the fact that these patients have a relatively short life and therefore less time for different factors to express their effects. However, there were also less patients in that age group and hence lower chance of reaching statistically significant results.

This study is remarkable for its large sample size and for the multitude of important covariates, for which the analyses were adjusted. This is also one of the first studies that evaluated the effects of recipient characteristics on outcomes separately in different age subgroups of senior patients in a large study population.

We also used a novel approach for transplant kidney survival analysis, that is, combined graft failure-imminent graft failure analysis. For kidney graft survival analysis, most studies perform unadjusted graft survival analysis, in which patient death is considered an endpoint for graft survival regardless of the functional status of the transplanted kidney at the time of death, and/or deathcensored graft survival approach, in which patients who die will be censored regardless of graft function at the time of death (25-28). In the deathcensored approach, patients with graft failure, who die before they have a chance to initiate another form of RRT, will not be considered as transplant failures. In the unadjusted approach, on the other hand, patient death is considered graft failure even if the transplanted kidney is functioning perfectly at the time of death. We defined the concept of "imminent graft failure" to include those who have not yet been started on another form of RRT, but their transplanted kidney is insufficiently functioning (Stage IV or more advanced CKD) and is imminent to become end stage. The combined graft failure-imminent graft failure approach provides a more accurate evaluation of longevity of the transplanted kidney and eliminates the mentioned potential errors incurred by the other two conventional methods.

Our study had some limitations. As with all registry-based observational studies, the current study suffered from certain limitations such as presence of missing data. Additionally, similar to all observational studies, the results cannot prove causality. Some information such as immunosuppressive and other therapeutic regimens, which have potential impacts on patient and graft survival, was not available in the SRTR database. The lower number of cases in the eldest subgroup of patients can potentially interfere with the statistical significance of the results. Furthermore, longer follow-up time could have provided more accurate information in terms of optimal use of transplanted kidney in the older recipient population as compared to younger recipients. Using angina as an indicator of coronary artery disease may not be inclusive; however, the SRTR database lacks information regarding the other indicators of coronary artery disease, which might be more accurate.

\section{Conclusions}

The number of senior patients with advanced CKD in need of RRT is growing rapidly. Kidney transplantation may attenuate the effect of aging on longevity. Moreover, advanced age is associated with relatively better kidney allograft outcomes (29). Additionally, most comorbidities are not associated with poorer outcomes in the oldest kidney transplant recipients ( $\geq 75 \mathrm{yr}$ old). Therefore, kidney transplantation should be encouraged for the appropriate senior patients. Additional studies of the potential disproportional protective effect of kidney transplantation on patient longevity may bring new insights into the management of kidney transplantation as well as the aging phenomenon and survival.

\section{Funding source}

The study was supported by KKZ's research grant from the American Heart Association grant (0655776Y). KKZ's other funding sources include the National Institute of Diabetes, Digestive, and Kidney Disease of the National Institute of Health (R01 DK078106); a research grant from DaVita Clinical Research; and a philanthropic grant from Mr. Harold Simmons. MZM is a recipient of the Hungarian Eötvös Scholarship (MÖB/77-2/2012).

\section{Acknowledgements}

The authors would like to thank DaVita Clinical Research (DCR) for providing patient data.

\section{References}

1. U.S. Census Bureau, Statistical Abstract of the United States: 2011. 
2. US Renal Data System: USRDS 2010 Annual Data Report: Atlas of Chronic Kidney Disease and End-Stage Renal Disease in the United States. Bethesda: National Institutes of Health, National Institute of Diabetes and Digestive and Kidney Diseases, 2010.

3. Giessing M, Budde K, Fritsche L et al. "Old-for-old" cadaveric renal transplantation: surgical findings, perioperative complications and outcome. Eur Urol 2003: 44: 701.

4. Johnson DW, Herzig K, Purdie D et al. A comparison of the effects of dialysis and renal transplantation on the survival of older uremic patients. Transplantation 2000: 69: 794.

5. Oniscu GC, Brown H, Forsythe JL. How great is the survival advantage of transplantation over dialysis in elderly patients? Nephrol Dial Transplant 2004: 19: 945.

6. Rao PS, Merion RM, Ashby VB, Port FK, Wolfe RA, KAYLER LK. Renal transplantation in elderly patients older than 70 years of age: results from the Scientific Registry of Transplant Recipients. Transplantation 2007: 83: 1069.

7. Wolfe RA, Ashby VB, Milford EL et al. Comparison of mortality in all patients on dialysis, patients on dialysis awaiting transplantation, and recipients of a first cadaveric transplant. N Engl J Med 1999: 341: 1725.

8. Gill JS, Tonelli M, Johnson N, Kiberd B, Landsberg D, Pereira BJ. The impact of waiting time and comorbid conditions on the survival benefit of kidney transplantation. Kidney Int 2005: 68: 2345.

9. Humar A, Denny R, Matas AJ, Najarian JS. Graft and quality of life outcomes in older recipients of a kidney transplant. Exp Clin Transplant 2003: 1: 69.

10. Pratschke J, Merk V, Reutzel-Selke A et al. Potent early immune response after kidney transplantation in patients of the European senior transplant program. Transplantation 2009: 87: 992.

11. Velez RL, Brinker KR, Vergne-Marini PJ et al. Renal transplantation with cyclosporine in the elderly population. Transpl Proc 1991: 23: 1749.

12. Morris GE, Jamieson NV, Small J, Evans DB, Calne R. Cadaveric renal transplantation in elderly recipients: is it worthwhile? Nephrol Dial Transplant 1991: 6: 887.

13. Doyle SE, Matas AJ, Gillingham K, Rosenberg ME. Predicting clinical outcome in the elderly renal transplant recipient. Kidney Int 2000: 57: 2144.

14. Saudan P, Berney T, Leski M, Morel P, Bolle JF, MarTIN PY. Renal transplantation in the elderly: a long-term, single-centre experience. Nephrol Dial Transplant 2001: 16: 824 .

15. Bentas W, Jones J, Karaoguz A et al. Renal transplantation in the elderly: surgical complications and outcome with special emphasis on the Eurotransplant Senior Programme. Nephrol Dial Transplant 2008: 23: 2043.

16. Oniscu GC, Brown H, Forsythe JL. How old is old for transplantation? Am J Transplant 2004: 4: 2067.

17. Fabrizi V, Winkelmayer WC, Klauser R et al. Patient and graft survival in older kidney transplant recipients: does age matter? J Am Soc Nephrol 2004: 15: 1052.

18. Kauffman HM, McBride MA, Cors CS, Roza AM, WYNN JJ. Early mortality rates in older kidney recipients with comorbid risk factors. Transplantation 2007: 83: 404.

19. Tesi RJ, Elkhammas EA, Davies EA, Henry ML, FerguSON RM. Renal transplantation in older people. Lancet 1994: 343: 461.

20. Heldal K, Hartmann A, Leivestad T et al. Clinical outcomes in elderly kidney transplant recipients are related to acute rejection episodes rather than pretransplant comorbidity. Transplantation 2009: 87: 1045.

21. Wu C, Shapiro R, Tan H et al. Kidney transplantation in elderly people: the influence of recipient comorbidity and living kidney donors. J Am Geriatr Soc 2008: 56: 231.

22. Tullius SG, Tran H, Guleria I, Malek SK, Tilney NL, Milford E. The combination of donor and recipient age is critical in determining host immunoresponsiveness and renal transplant outcome. Ann Surg 2010: 252: 662.

23. Molnar MZ, Huang E, Hoshino J et al. Association of pretransplant glycemic control with posttransplant outcomes in diabetic, kidney-transplant recipients. Diabetes Care 2011: 34: 2536.

24. Prevention CfDCa. Life expectancy at birth, at 65 and 75 years of age by race and sex Health, United States 2010. 2010.

25. Chakkera HA, O'Hare AM, Johansen KL, Hynes D, Stroupe K, Colin PM, Chertow GM. Influence of race on kidney transplant outcomes within and outside the Department of Veterans Affairs. J Am Soc Nephrol 2005: 16: 269.

26. Tullius SG, Tilney NL. Both alloantigen-dependent and -independent factors influence chronic allograft rejection. Transplantation 1995: 59: 313.

27. Weiss AS, Smits G, Wiseman AC. Twelve-month pancreas graft function significantly influences survival following simultaneous pancreas-kidney transplantation. Clin $\mathbf{J}$ Am Soc Nephrol 2009: 4: 988.

28. Hariharan S, Johnson CP, Bresnahan Ba, Taranto SE, McIntosh MJ, Stablein D. Improved graft survival after renal transplantation in the United States, 1988 to 1996. N Engl J Med 2000: 342: 605.

29. Molnar MZ, Streja E, Kovesdy CP et al. Age and the associations of living donor and expanded criteria donor kidneys with kidney transplant outcomes. Am J Kidney Dis 2012: 59: 841.

\section{Supporting Information}

Additional Supporting Information may be found in the online version of this article:

Figure S1. Distribution of age in 15667 kidney transplant patients $65 \mathrm{yr}$ or older.

Table S1. Relative risk of death in different age groups in kidney transplant recipients and the general population.

Table S2. Hazard ratios (95\% confidence intervals) of death-censored graft failure and combined graft failure using Cox regression analyses in 10101 kidney transplanted patients aged between $65-<70$ years.

Table S3. Hazard ratios (95\% confidence intervals) of death-censored graft failure and combined graft failure using Cox regression analyses in 4271 kidney transplanted patients aged between $70-<75$ years.

Table S4. Hazard ratios (95\% confidence intervals) of death-censored graft failure and combined graft failure using Cox regression analyses in 1295 kidney transplanted patients aged $\geq 75$ years. 